\title{
IMS at the crossroads
}

\section{The success of next week's ISEE satellite mission is crucial to the IMS. Judy Redfearn outlines why}

PART of the International Sun-Earth Explorer (ISEE), the second major satellite mission in the International Magnetospheric Study (IMS), was due for launch this week aboard one of NASA's Delta launch vehicles. But because of delays to NASA's launch programme caused by the failure last month of the Delta rocket carrying the European Space Agency's (ESA) Orbital Test Satellite (OTS), the ISEE launch has been put back, probably until 19 October. If the launch fails to go ahead then, or at least by the end of the month, it will face a lengthy hold-up because the current period of short daily launch windows will then have passed by; the ISEE mission would have to remain earthbound until next spring.

Magnetospheric scientists will be watching the launch with a degree of apprehension for another reason. Earlier this year, a Delta launcher failed to send Europe's Geos, the first IMS satellite mission, into geostationary orbit. Although the consequences are not as severe as was first thought, the IMS programme has suffered a setback. So the success of ISEE is now more important than ever to the success of the IMS. If all goes well next week, a unique programme will begin to find out the precise mechanisms governing the magnetosphere, the area of the near-earth environment where the solar wind interacts with the earth's magnetic field.

For the first time two satellites, NASA's ISEE A and ESA's ISEE B, are to be launched in tandem aboard a Delta 2914 rocket into the same highly elliptical orbit. ISEE B, the 'daughter', will follow ISEE $\mathrm{A}$, the 'mother', at a distance varying between $100 \mathrm{~km}$ and $5,000 \mathrm{~km}$. The satellites will carry carefully matched experiments allowing them both to make comparable measurements of the same phenomena. Because they will be moving at a known speed and distance apart, they will be able to distinguish between transient phenomena and those which are permanent but vary in space. A single satellite suffers from not knowing whether a phenomenon has moved past it or has died out.

\section{Crossing boundaries}

Although many phenomena are known to exist in the magnetosphere, they are not well understood because of the problem of distinguishing spatial from temporal variations. In particular little is known about the processes which occur at the boundary where the solar wind meets the magnetosphere. The highly elliptical orbit of ISEE A and B was designed to maximise the number of crossings of this boundary, or bowshock, during the satellites' three-year lifetime. The orbit chosen has a 59 . hour period with a closest distance from earth of $280 \mathrm{~km}$ and a furthest distance of about $140,000 \mathrm{~km}$. It will also carry the satellites through other boundaries, such as the magnetopause, plasmapause and neutral current sheet.

In June next year, ISEE $A$ and $B$ will be joined by the third and final part of the ISEE mission, ISEE C. Unlike $A$ and $B, C$ will be launched into an orbit around the sun at a distance of about 1.6 million $\mathrm{km}$ from the earth. For fuel economy, the orbit was chosen to lie on a libration point where the gravitational forces between the sun, earth and moon almost cancel out.

While $\mathbf{A}$ and $\mathbf{B}$ are measuring processes in the magnetosphere, from its position in deep space $C$ will be monitoring variations in the solar wind and sending information on solar particles back to earth an hour in advance of the particles reaching earth themselves. The data will give a picture of the solar wind which will help in understanding observations made by the $\mathbf{A}$ and $\mathbf{B}$ satellites.

At the end of the mission, scientists should know a lot more about the solar wind and the processes governing the magnetosphere, which may in turn lead to a better understanding of radio transmission on earth and some of the mechanisms effecting climate. The magnetosphere, a natural plasma laboratory surrounding the earth, is like the earth's own small universe, the solar wind taking the part of the hot plasma which makes up most of the matter in space and the earth's magnetic field taking the part of the magnetic fields found in space. The study of how ionised particles interact with the earth's magnetic field should yield much about the fundamental processes which govern the behaviour of cosmological objects such as radiogalaxies, pulsars and radio and X-ray stars.

\section{Setting up the IMS}

By the late 1960 s scientists had a good picture of the magnetosphere, but they knew little of the dynamic processes controlling it. The International Magnetospheric Study was set up as a coordinated attack on the remaining problems. To organise the study, the International Council of Scientific Unions (ICSU) Special Committee on Solar-Terrestrial Physics set up an IMS steering committee with representatives from various scientific unions, COSPAR and satellite launching agencies. Thirty-nine countries are now taking part in the IMS, which began in 1976 and is due to run until December 1979.

It is the first international programme of its kind to be based on spacecraft flown by several different agencies. Fourteen spacecraft launched during the IMS period are planned to carry experiments directly relevant to the study and 25 launched before 1976 are expected to add valuable data. But at the centre of the IMS are two spacecraft missions devoted entirely to magnetospheric science-Geos and the ISEE. In addition, Japan is contributing with its magnetospheric satellite, Exos, and Russia with a near-earth satellite.

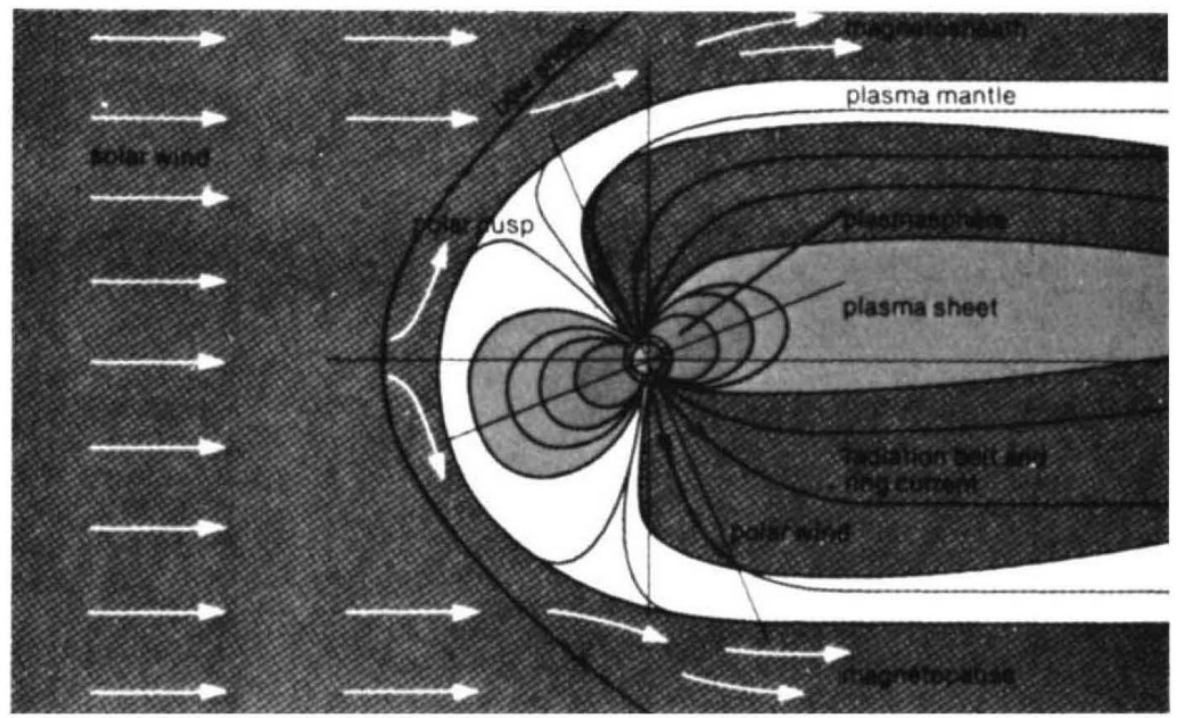

Cross-section of magnetospheric plasma distribution 
The spacecraft programme, however, would be of little value without the numerous ground-based rocket and balloon projects which will continue throughout the IMS period. Earthbased measurements of variations in the magnetic field can be used to monitor magnetic storms and plasma waves and even to predict changes in the magnetosphere. Correlating these measurements with satellite observations should indicate how changes in field strength affect the shape and dynamics of the magnetosphere. Many of the 39 countries which could not afford to be involved in the space programme are contributing to the IMS through a sophisticated world-wide network of ground observatories. Even at the beginning of 1976, more than 1,000 individual research projects were registered on the IMS computerised file.

Many different experimental programmes working towards the same end thus need coordinating. Occasionally, for example, two or more satellites may be in such a configuration that simultaneous observations would be very valuable. Similarly, satellite observations should sometimes be timed in with ground-based or rocket experiments. To coordinate observations, NASA helped set up an IMS Satellite Situation Centre at the beginning of the study at the World Data Center A for Rockets and Satellites at the Goddard Space Flight Center, Boulder, Colorado. Its function is to report on satellite positions, recommend periods of special interest, compile and up-date information on satellite experiments and answer inquiries on special projects, experiments and the whereabouts of satellites. Every month, the IMS Central Information Exchange Office, also at Boulder, produces an IMS news letter.

\section{Regaining ground}

Hopes for regaining the ground lost by the partial failure of Geos hang on the possibility of launching the second Geos satellite into geostationary orbit carly next year. The intention had been to place the first Geos in a geo-

International science (2)

\section{WHO's show on the road}

\section{Peter Collins describes the gestation of the World Health Organisation's tropical diseases progamme}

\begin{abstract}
$T^{\mathrm{HE}}$ temptation to launch global 1 campaigns against specific diseases or conditions of mankind is one the World Health Organisation (WHO) seems unable to resist. The most recent and from all appearances most successful is the smallpox eradication campaign. Pockets of the disease remain, principally in the horn of Africa, but the campaign's objectives are in sight in under ten years. By contrast, in spite of the malaria eradication campaign, which started in 1955, malaria has neycr been anywhere near eradication in much of Africa south of the Sahara, and is now increasing in other regions and countries where eradication was thought to have been achieved. Now WHO's thinking has turned towards a more broadly-based approach. It is well exemplified by the Special Programme on Research and Training in Tropical Diseases which is tackling six diseases (malaria, schistosomiasis, the filariases, trypanosomiasis, Leishmaniasis and leprosy) that affect some 700 million men, women and children.
\end{abstract}

Perhaps the greatest single constraint on the development of the tropics lies in the burden of disease borne by their human inhabitants. Probably one in every four people alive today is at risk from one or more of the six diseases with which the programme is concerned. The position worsens every year as population increases and the world's pharmaceutical industry stands back. No new drug against trypanosomiasis has appeared in the past 20 years; the only drugs available against the filariases have side effects so serious that their use for mass treatment is out of the question; and the drugs used for treating malaria are meeting with more and more resistance on the part of the parasites. Added to this is the increasing resistance of many of the vectors of these diseases to the chemicals used against them, and the many reservations about the use of DDT, still often the most effective weapon for insect vector control.

The sheer lack of scientific knowledge about many of these diseases is also a problem. Not enough is known about the biology of the parasite and the mechanisms by which it eludes the defences of the human host to allow intelligent intervention to be planned. But great advances in such fields as immunology have led to the evolution of a whole armoury of new weapons for the prevention, diagnosis and treatment of disease afflicting richer stationary orbit $36,000 \mathrm{~km}$ above the equator along a field line connecting the north and south auroral zones so that particles on their way to the aurora would pass through it. Groundbased experiments and rocket-borne observations of the aurora could then have been coordinated with experiments on board Geos.

But Geos is not in a geostationary orbit and coordinating observations is very difficult. It would have been impossible had the Geos ground control at Darmstadt not managed to manoeuvre the satellite into a doubly geosynchronous orbit shortly after the unsuccessful launch. Geos now passes through the neighbourhood of the planned geostationary orbit twice a day, meaning that coordinated observations are possible, but only for a very short time. If Geos 2 were launched next year, it could take the place of Geos 1. It would have to be launched on another Delta rocket, however, and ESA might decide to wait until the end of 1979, when it could be launched on Europe's launcher, Ariane.

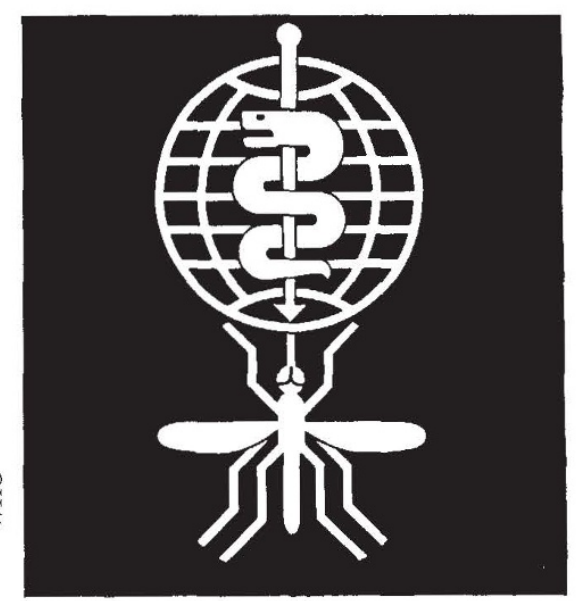

WHO anti-malaria emblem

countries, and the question is how these can be made available or adapted to help control the great endemic diseases of the tropical countrics.

\section{WHO's answer}

The present programme is WHO's answer to this question, but it originated within WHO from the conjunction of two quite scparate idcas. In 1964 an expert group of immunologists and parasitologists was assembled for the first time in Geneva. Little common ground seemed to exist between these two disciplines thereto: the parasitologists considered that there was little if any immune response to the diseases with which they were concerned, and the immunologists had found ample scope, backed by adequate funds, in fields like cancer research. After this meeting, however, things began to change, and while the immunology of 